\title{
Virtual reality inspection and painting with measured BRDFs
}

Dal Corso, Alessandro; Stets, Jonathan Dyssel; Luongo, Andrea; Nielsen, Jannik Boll; Frisvad, Jeppe Revall; Aanæs, Henrik

\section{Published in:}

Proceedings of SA '17 VR Showcase

Link to article, DOI:

$10.1145 / 3139468.3139472$

Publication date:

2017

Document Version

Peer reviewed version

Link back to DTU Orbit

Citation (APA):

Dal Corso, A., Stets, J. D., Luongo, A., Nielsen, J. B., Frisvad, J. R., \& Aanæs, H. (2017). Virtual reality inspection and painting with measured BRDFs. In Proceedings of SA '17 VR Showcase Association for Computing Machinery. https://doi.org/10.1145/3139468.3139472

\section{General rights}

Copyright and moral rights for the publications made accessible in the public portal are retained by the authors and/or other copyright owners and it is a condition of accessing publications that users recognise and abide by the legal requirements associated with these rights.

- Users may download and print one copy of any publication from the public portal for the purpose of private study or research.

- You may not further distribute the material or use it for any profit-making activity or commercial gain

- You may freely distribute the URL identifying the publication in the public portal 


\section{Virtual Reality inspection and painting with measured BRDFs}

\author{
Alessandro Dal Corso \\ Technical University of Denmark \\ Jannik Boll Nielsen \\ Technical University of Denmark
}

\author{
Jonathan Dyssel Stets \\ Technical University of Denmark \\ Jeppe Revall Frisvad \\ Technical University of Denmark
}

\author{
Andrea Luongo \\ Technical University of Denmark \\ Henrik Aanæs \\ Technical University of Denmark
}
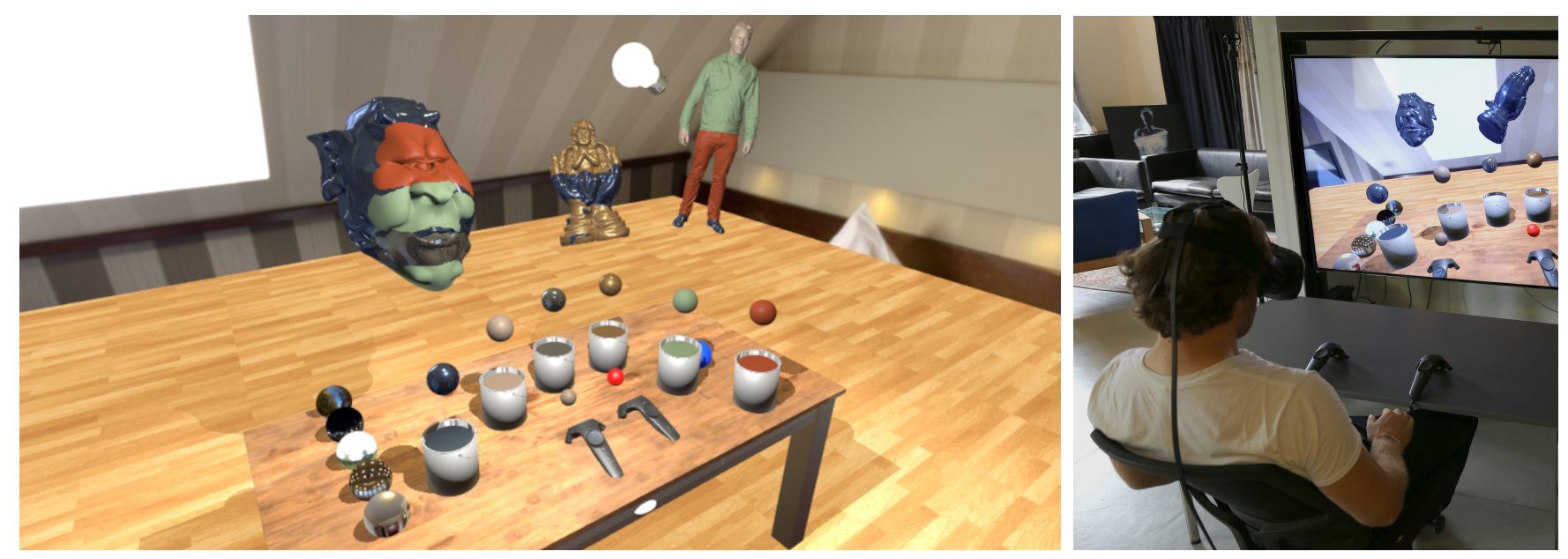

Figure 1: Pictures illustrating our VR demo application, with an in-game screenshot (left) and a picture of the setup (right). Paintbucket, table and lightbuld models $\odot$ TurboSquid.com, environment maps $\odot$ HDRMaps.com and $\odot$ Joost Vanhoutte.

\section{CCS CONCEPTS}

- Computing methodologies $\rightarrow$ Virtual reality;

\section{KEYWORDS}

Virtual Reality, material appearance

\section{ACM Reference Format:}

Alessandro Dal Corso, Jonathan Dyssel Stets, Andrea Luongo, Jannik Boll Nielsen, Jeppe Revall Frisvad, and Henrik Aanæs. 2017. Virtual Reality inspection and painting with measured BRDFs. In Proceedings of SA '17 VR Showcase, Bangkok, Thailand, November 27-30, 2017, 2 pages.

https://doi.org/10.1145/3139468.3139472

\section{INTRODUCTION}

This is a virtual reality (VR) painting application that enables the user to paint on 3D models with real measured materials, much like in the physical world. A scanned physical object can be imported into the VR application, and the user can paint on the surface of the object with a virtual hand-controlled paint brush. The user is presented with several paint buckets, each containing a material known from the physical world. These materials are measured bidirectional reflectance distribution functions (BRDFs) of real physical

SA '17 VR Showcase, November 27-30, 2017, Bangkok, Thailand

(C) 2017 Copyright held by the owner/author(s).

This is the author's version of the work. It is posted here for your personal use. Not for redistribution. The definitive Version of Record was published in Proceedings of SA '17 VR Showcase, November 27-30, 2017, https://doi.org/10.1145/3139468.3139472. materials. The materials and objects present in VR are thus represented as they would be in the physical world, and the user can control both the environment lighting and a single light source. The application enables analog artists to apply their skills directly on a digital model and it enables engineers to directly inspect BRDFs in a fast and intuitive way. Figure 1 is a screenshot from the application showing models that have been painted with BRDFs.

\section{MOTIVATION}

The realization of our VR demo was mainly driven by two objectives. The first was to have an intuitive and practical way of inspecting and visualizing measured BRDFs. Our application enables the user to apply BRDFs to 3D objects in a simple way that mimics the action of painting in real life. The user can also interact with the environment and the objects through an intuitive interface. Some of the actions the user can perform consist of modifying the light source intensity and position, the environment map, and the position and orientation of the objects. All these features allow us to quickly visualize and inspect a chosen BRDF under different lighting conditions without having to interact with a complicated interface.

Our second objective was to have an application useful in industrial and artistic design [Wald et al. 2006]. We provide a new way for artists, both digital and analog, to transfer their skills to the 3D domain by painting materials directly on 3D models that they might have created or scanned from real objects. By providing a real-time rendering environment, we enable artists to immediately see the final results of their creations under different viewing perspectives and lighting conditions. With this VR application, we aim at moving 


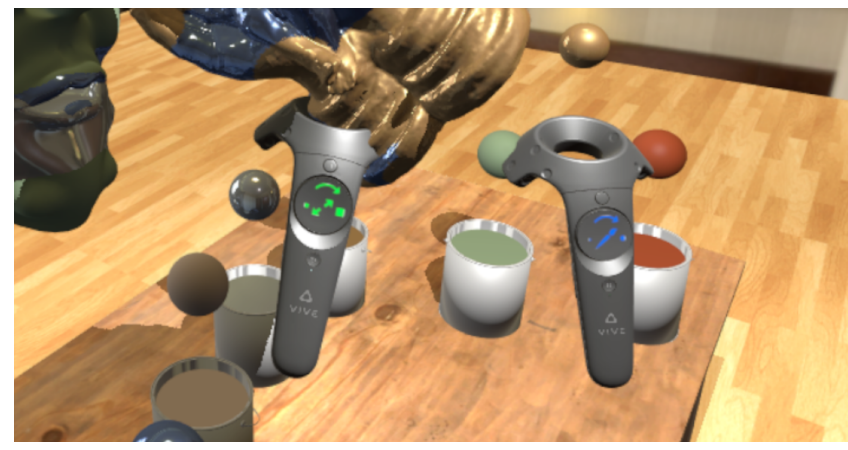

Figure 2: Interaction (left) and paintbrush (right) controller. Note the icons allowing to change the model and the brush size, respectively. See Figure 1 for copyright notice.

a first step towards the creation of a tool that will allow artists to apply materials and colors in a way that is similar to what they would do in the real world. Thus, we support the more traditional artistic workflow rather than inventing new artistic workflows for editing BRDFs [Colbert et al. 2006].

A user entering the virtual world will see a simple scene: a table, calibrated to match the position and height of a table present in the real world, a set of paint buckets containing different materials based on measured BRDFs, several 3D models, and a light-bulb. The user interacts with the scene through two handheld trackable controllers, one used as a painting tool and the other one as a grabbing and interaction tool. $\mathrm{He} /$ she can grab, move, rotate, and scale the 3D models with one hand and paint and select a different material with the other. The user can also move the position of the light-bulb and its intensity to get an immediate feedback on the appearance of their work. Furthermore, we use haptic feedback to enhance the interaction between user and objects in the scene. Our application thus creates a bridge between the digital and the physical domain, with an interface known from the physical world that the user is already familiar with. All these features help users immerse themselves and unfold their creativity in an environment similar to what they would see in a real artist's studio, and we have aimed to make this environment as interactive as possible.

\section{DETAILS}

Our demo is an HTC Vive setup (https://www.vive.com/), using the two provided controllers as interaction devices. One of the controllers is used for interaction, while the other acts as a paintbrush. The interaction is activated using the trigger button on the controller, and is used for grabbing and moving objects, including the light bulb above the table and the paint buckets. We use the small spheres on the left side of the table to change environment map. Finally, we have an undo button on the right to undo/redo the last action performed. Dipping the paintbrush into a bucket changes the BRDF that it will paint with. Based on the controller touchpads (see Figure 2), we also added interactions for changing object size (while grabbed), paintbrush size (on the paintbrush controller), and light intensity (while the light is grabbed).

We use measured BRDFs both from the MERL database [Matusik et al. 2003] and from our own laboratory. The scene has two light sources: an environment map and a movable point light in the form of a light bulb. The environment map contributes with background, reflections, and an ambient term. The ambient term is computed through standard spherical harmonics multiplied by the bihemispherical reflectance $\rho$ of each measured BRDF, calculated in a preprocessing step using Monte Carlo integration. We multiply the environment map reflected color by $\rho$ and by the factor $\min \left(1, \frac{f_{\max }}{C \rho_{\text {avg }}}-1\right)$, where $f_{\max }$ is the peak value in the measured material, $\rho_{\text {avg }}$ is the average of the three channels of $\rho$, and $C$ is a user-defined constant. In case of a material with a strong reflection peak (such as a metallic paint), $\rho_{\text {avg }} \ll f_{\max }$ and the factor will be equal to one. In a case of a more diffuse material, $\rho_{\text {avg }} \approx f_{\max }$ and the reflection term will not be included. To paint the material, we intersect a sphere with the vertices of the model. For materials without a UV map, we write a material label on a per-vertex data structure. If a UV map is present, we first generate a secondary texture that maps vertex coordinates to UVs and then write the labels into a texture using the generated mapping.

\section{USER FEEDBACK AND CONCLUSION}

We invited an analog artist, a design engineer, and two 3D artists to test our application. They all found the interface intuitive to work with. Most noticeably, the analog artist used the full system without any previous VR experience after a one minute verbal instruction. This supports the objective of our application to enable transfer of artistic skills from the analog to the digital domain. Users noted the bulkiness of the controller compared to real-life painting tools such as a brush or a pencil. We accept this limitation of the system, hoping for smaller, lighter, or more customizable solutions in the future, like the stylus presented by Jackson and Keefe [2016].

Our VR painting application enables the user to paint on 3D models with measured BRDFs. Our users praised how our application is intuitive to use, and how it creates a bridge between the analog and digital skills. Furthermore, it enables a fast and intuitive way to inspect BRDFs under various lighting conditions.

\section{Acknowledgements}

Paint bucket, table, and light bulb models are courtesy of TurboSquid.com. Environment maps: studio, garden, and sunset forest from hdrmaps.com, lobby and night city square from Joost Vanhoutte. We use chrome, blue paint, gold paint and red fabric BRDFs from the MERL database. Ogre model courtesy of Keenan Crane. VIVE and related assets are property of HTC, Inc. and Valve Corporation. We would like to thank users Felicia Frisvad, Jon Murray Vinther, Sam Surplice, Christian Ahm for their valuable feedback.

\section{REFERENCES}

M. Colbert, S. Pattanaik, and J. Krivanek. 2006. BRDF-Shop: creating physically correct bidirectional reflectance distribution functions. IEEE Computer Graphics and Applications 26, 1 (Jan 2006), 30-36. https://doi.org/10.1109/MCG.2006.13

B. Jackson and D. F. Keefe. 2016. Lift-off: Using reference imagery and freehand sketching to create 3D models in VR. IEEE Transactions on Visualization and Computer Graphics 22, 4 (2016), 1442-1451. https://doi.org/10.1109/TVCG.2016.2518099

W. Matusik, H. Pfister, M. Brand, and L. McMillan. 2003. A Data-Driven Reflectance Model. ACM Transactions on Graphics 22, 3 (July 2003), 759-769.

I. Wald, A. Dietrich, C. Benthin, A. Efremov, T. Dahmen, J. Gunther, V. Havran, H. p. Seidel, and P. Slusallek. 2006. Applying Ray Tracing for Virtual Reality and Industrial Design. In 2006 IEEE Symposium on Interactive Ray Tracing. 177-185. https://doi.org/10.1109/RT.2006.280229 